\title{
Experimental study on damage identification for grid structure based on
}

\section{BP neural network}

\author{
Xing Zhe ${ }^{1, a}$, Yang Chun-he ${ }^{2, b}$, Yang Bin $^{3, c}$ \\ ${ }^{1}$ Civil Engineering College, Tongji University, Shanghai, China \\ ${ }^{2}$ Civil Engineering College, Tongji University, Shanghai, China \\ ${ }^{3}$ Civil Engineering College, Tongji University, Shanghai, China \\ a1530671@tongji.edu.cn, b1630388@tongji.edu.cn, cyangbin@tongji.edu.cn
}

Key words: damage identification; modal parameter; BP neural network

Abstract: Aiming at the difficulties of modal concentration and high degree of freedom in the damage identification of the truss structure and the good fault tolerance and robustness of the BP network, based on the theory of the change of the modal parameters of the truss structure before and after the damage, the modal parameters and BP neural Network structure damage identification method. Taking a $6 \mathrm{~m} \times 7.5 \mathrm{~m}$ square pyramidal grid structure as the research object, the importance coefficient of each bar was calculated according to the theory of continuous collapse, and the position of the damaged bar was simulated. Then, the square of the normalized frequency of the structure before and after damage And the combination of normalized vibration mode parameters as damage indicators to train, test and test BP neural network. The results show that this method can well identify the location and extent of damage to the grid structure.Damage identification, including damage judgment, location and degree, is one of the core of SHM ${ }^{[1]}$. The change of modal parameters before and after the damage can be regarded as the sign of structural damage to diagnose the position and degree of structural damage.

\section{BP neural network on damage identification}

Neural network is a network system that imitates the mechanism of human brain information processing. Neural network is widely used in many fields such as civil engineering. ${ }^{[2]}$ The nature of damage identification by neural networks is to establish the nonlinear mapping relationship between input (structural characteristics) and output (damage judgment, location and degree).

The earliest use of BP neural network for damage identification is Venkatasubramanian and $\mathrm{Chan}^{[3]}$ from Purdu University in the United States. In 1989, they first used BP network to identify damage for large structures. Next many scholars at home and abroad are committed to this study. $\mathrm{Wu}^{[4]}$ used 200 discrete spectral points as the input of BP neural network to identify the damage of a three-layer shear-frame model and identify the damage from the measured structural response. Yang $\mathrm{Na}^{[5]}$ et al. combined the neural network and finite-element model correction method, the damage identification of typical Tibetan structures has made more real results. Wu Jinzhi ${ }^{[6]}$ combined the dynamic characteristics of the grid structure, put forward the damage characteristic parameters - vibration mode vector and the rate of change of frequency combination parameters.

On the basis of this study, a $6 \mathrm{~m} \times 7.5 \mathrm{~m}$ grid structure of scale-square square pyramid space grids was designed to simulate the damage manually. The modal parameters of the structure under various damage conditions were obtained. The structural normalized frequencies squared change rate and normalized displacement mode as the damage index, BP neural network is used to identify 
the damage step by step, and more meaningful research results have been obtained.

\section{Square pyramid space grids structure experimental design}

\section{structure model}

The experimental structure is a square pyramid space grids model. Grid length and short dimensions are $7.5 \mathrm{~m}$ and $6 \mathrm{~m}$, and arrow height is $1 \mathrm{~m}$. Wound length of 5 cross, short to 4 cross, the lower chord length 4 cross, short to 3 cross, the grid size of $1.5 \mathrm{~m} \times 1.5 \mathrm{~m}, 4$ fixed bearings, and the node bolted ball connection. String abdominal rod specifications are $\varphi 48 \times 3.5$, and the material is Q345, bolt ball $\varphi 100$. A total of 50 nodes, 160 bar unit, test structure shown in Figure 1.

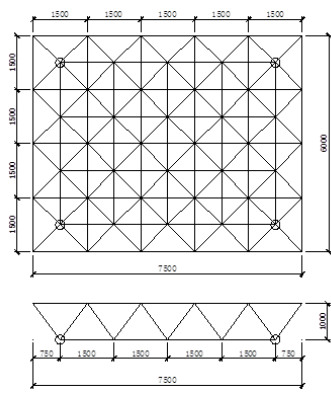

Fig.a Structural layout plan
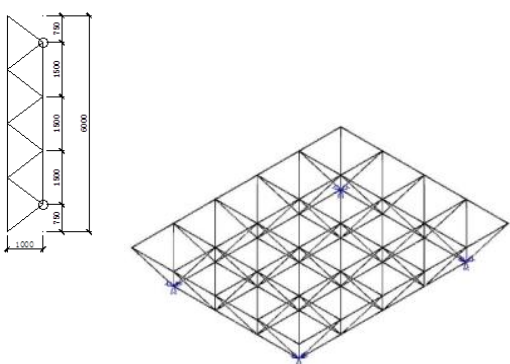

Fig.b Graphic model

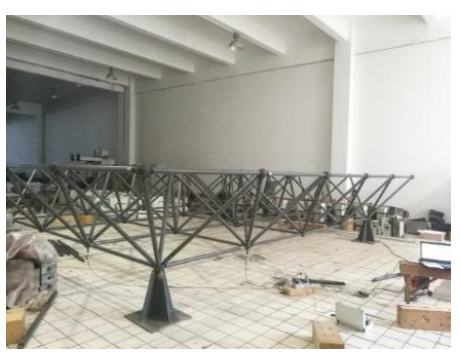

Fig.c Experimental grid

\section{Design of test grid damage condition}

Fig.1 Experimental grid structure

The test of the injury program designed to:

(1) Local damage to the rod $1 / 3$, the specific approach is: a long $300 \mathrm{~mm}$, into the depth of $12 \mathrm{~mm}$ section of the pipe wall, after processing the damaged rod shown in Figure 2;

(2) The instantaneous failure of the bar member is performed by wire cutting in the middle of the bar member to form a jagged Steel head, before simulation failure, the normal compressive stress to the steel head is provided by the fixture, the fixture is released and the rod is separated, so as to realize the instant failure of the rod. Processed damaged rod is shown in Figure 3.

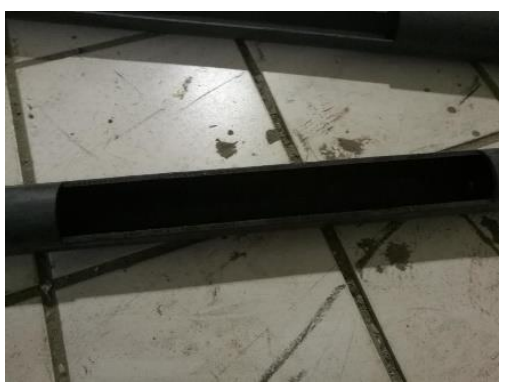

Fig.2 Local damaged member

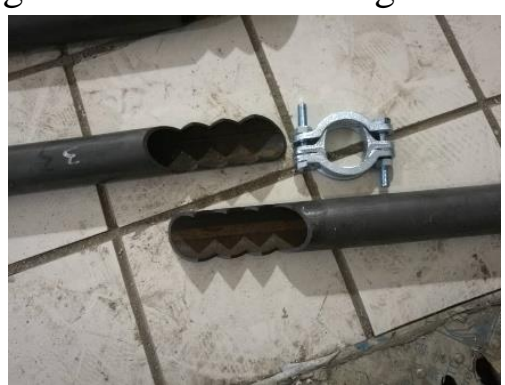

Fig.3 Suddenly failure member

The selection of simulated damage location combined with the theory of continuous collapse.The failure will cause the structure of the local body of the rod as the calculation object, select the buckling coefficient, stress, total strain energy, the rod strain energy as a sensitive parameter, normalized and average, the rod number shown in Figure 4.

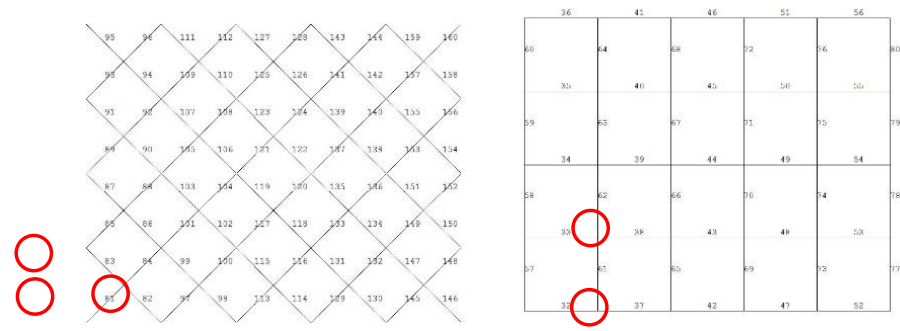

Fig.4 Members' number and important members 
In addition to undamaged condition, the test take the rod number 42, 43, 82, 84, 98 respectively, $33 \%, 100 \%$ degree of stiffness damage, a total of 11 kinds of test conditions.

\section{BP neural network on damage identification}

\section{BP neural network input and output parameters}

Before generating structural damage pattern samples, it is necessary to determine the input parameters of the network. This parameter not only affects the operation of the network, but also affects the generalization ability of the network. In this paper, the combination of square rate of change of normalized frequency and normalized mode of vibration is selected as the input parameter of damage identification network.

$$
\left\{F S C R_{1}, \ldots, F S C R_{m}, D F_{1}, \ldots, D F_{n}\right\}
$$

Where $\mathrm{m}$ is the order of the vibration mode used, $\mathrm{n}$ is the frequency order used.

$F S C R_{i}$ is regularized frequency square rate of change.

$D F_{i}$ is the i mode modal corresponds to the normalized mode vector of $\mathrm{p}$ test degrees of freedom.

The network training samples are numerically analyzed, and the model is established by ANSYS software.

\section{BP neural network training and testing}

The undamaged and damaged samples of 42, 43, 82, 84 and 98, respectively, with damage of $25 \%, 50 \%, 75 \%$ and $100 \%$, were used to establish the learning samples of BP neural network with a total of 21 groups Data to participate in network training. The structure of the network is composed of 8 input layer neurons, 17 hidden layer neurons and 5 output layer neurons. In each unit, when the rigidity damage is $33 \%$ and $100 \%$, respectively, a total of 10 conditions The damage index (part of the data in Table 1) obtained by the experiment is used as a test sample of BP neural network to test the anti-noise performance and generalization ability of the network. Part of the test results shown in Figure 5.

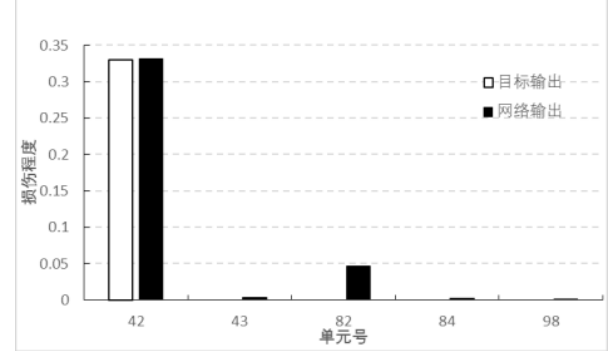

Fig.a NO.42 33\% degree of damage

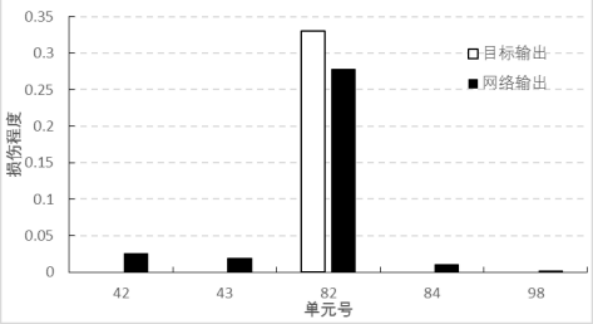

Fig.c NO.82 33\% degree of damage

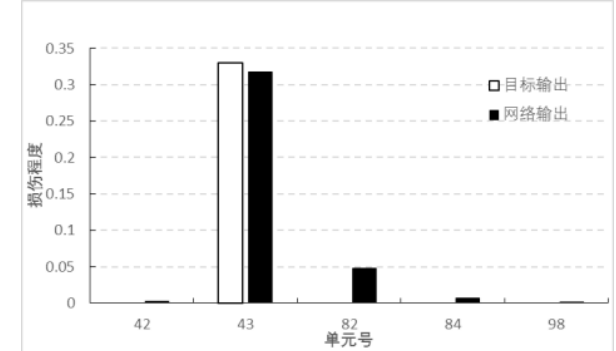

Fig.b NO.43 33\% degree of damage

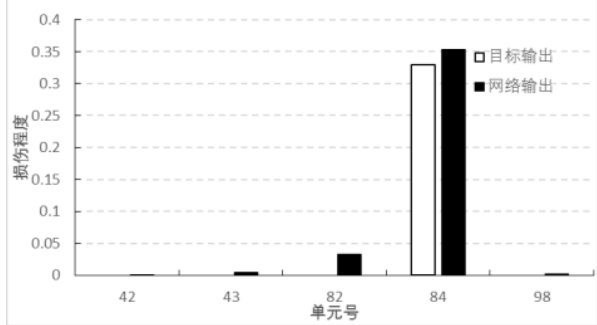

Fig.d NO.84 33\% degree of damage

Fig.5 Results of damage identification 
Tab.1 Testing result of BP network

\begin{tabular}{cccc|cccc}
\hline No. & $\begin{array}{c}\text { Target } \\
\text { output }\end{array}$ & $\begin{array}{c}\text { Bpnn } \\
\text { output }\end{array}$ & error & No. & $\begin{array}{c}\text { Target } \\
\text { output }\end{array}$ & $\begin{array}{c}\text { Bpnn } \\
\text { output }\end{array}$ & error \\
\hline 42 & 1 & 0.9737 & $-2.63 \%$ & 42 & 0.33 & 0.3311 & $0.34 \%$ \\
43 & 1 & 0.9866 & $-1.34 \%$ & 43 & 0.33 & 0.3176 & $-3.75 \%$ \\
82 & 1 & 0.9802 & $-1.98 \%$ & 82 & 0.33 & 0.2775 & $-15.92 \%$ \\
84 & 1 & 0.9981 & $-0.19 \%$ & 84 & 0.33 & 0.3533 & $7.05 \%$ \\
98 & 1 & 0.9811 & $-1.89 \%$ & 98 & 0.33 & 0.3721 & $12.75 \%$ \\
\hline
\end{tabular}

\section{Conclusion}

(1) The results show that the BP neural network has good fault-tolerance and robustness. In the presence of experimental error, the identification of the single damage of the structure has achieved a good recognition effect.

(2) The combination of the squared rate of change of the standardization frequency and the normalized mode of displacement proposed in this paper can well serve as the damage index of the truss structure. Considering the test conditions and some precision requirements, use the low-order frequency as a damage indicator is feasible.

(3) In the simulation of damage conditions, the damaged elements are selected to simulate the damaged elements in combination with the continued failure theory, so the damaged conditions are representative.

\section{Acknowledgements}

This work was financially supported by the National Natural Science Foundation of China (No. 51408432).

\section{References}

[1] Sun Hong-min, Li hong-nan. Progress in Health Monitoring of Civil Engineering Structures[J]. Journal of Disaster Prevention and Mitigation Engineering, 2003, 23(3): 92-98.

[2] Xiong Zhong-ming, Wang Chao, Lin Tao. Defect determination of Large-span Steel Structure Based on Neural Network[J]. Journal of Vibration and Shock, 2011,30(9): 191-196.

[3]Venkatasubramanian V and Chan K. A neural network methodology for process fault diagnosis[J]. Journal of AIChE， 1989， 35(12): 1993-2002.

[4] X.Wu, J. Ghaboussi and J.H. Garrertt. Use of Neural Networks in Detection of Structural Damage[J]. Computers and Structures. 1992, 42(4):649-659.

[5] Yang Na, Zhang Yan. Finite Element Model Updating of Tibetan Structure Based on Artificial Neural Network[J]. Journal of Vibration and Shock, 2013, 32(9):125-129.

[6] Wu Jin-zhi. Researh on Damage Identification of Grid Structures Based on the Vibration Parameters[D]. Beijing: Beijing University of Technology, 2005. 